\title{
Direct observations of the photoinduced change in dimerization in K-TCNQ
}

\author{
Tadahiko Ishikawa, ${ }^{1, *}$ Ryosuke Hosoda, ${ }^{1}$ Yoichi Okimoto, ${ }^{1}$ Sei' $i c h i$ Tanaka, ${ }^{1}$ Ken Onda, ${ }^{2,3}$ \\ Shinya Koshihara, ${ }^{1}$ and Reiji Kumai ${ }^{4}$ \\ ${ }^{1}$ Department of Chemistry and Materials Science, Tokyo Institute of Technology, 2-12-1, Oh-okayama, Meguro-ku, Tokyo, 152-8551, Japan \\ ${ }^{2}$ Interactive Research Center of Science, Tokyo Institute of Technology, Nagatsuta, Midori-ku, Yokohama, Kanagawa 226-8502, Japan \\ ${ }^{3}$ PRESTO, Japan Science and Technology Agency (JST), 4-1-8 Honcho, Kawaguchi, Saitama 332-0012, Japan \\ ${ }^{4}$ Condensed Matter Research Center and Photon Factory, Institute of Materials Structure Science, KEK, Tsukuba, 305-0801, Japan
}

(Received 8 December 2015; revised manuscript received 29 April 2016; published 17 May 2016)

\begin{abstract}
The photoinduced dynamics of a potassium-tetracyanoquinodimethane (K-TCNQ) single crystal in the generalized Peierls phase are evaluated via time-resolved vibrational spectroscopy. The transient reflectivity spectrum of the photoinduced state in the mid-IR range shows a decrease in the height and width of the reflectivity band because of the electron-molecular-vibration-coupled $\mathrm{CN}$ stretching mode at approximately $2180 \mathrm{~cm}^{-1}$. This spectral change suggests that the photoexcitation of the charge transfer in TCNQ molecules induces melting of the dimerization of the molecules. From detailed analysis of the spectral evolution, the relaxation time constant from the photoinduced state to the dimerized state is estimated to be approximately $0.6 \mathrm{ps}$. Even after the recovery of the dimerization, a fluctuation is still observed, probably because of a domain-wall soliton. The fluctuation gradually dissipates with a time constant of approximately $2.3 \mathrm{ps}$. Direct observation of the dimerization process reveals the true dynamics of the photoinduced cooperative phenomenon within this system.
\end{abstract}

DOI: 10.1103/PhysRevB.93.195130

\section{INTRODUCTION}

Light excitation of electrons in crystalline solids may induce macroscopic changes by virtue of the cooperative interactions inherent within the system. This phenomenon has attracted much interest and is called photoinduced phase transition (PIPT) [1]. Quasi-one-dimensional (1D) molecular crystals are excellent candidates of a system where highly efficient PIPT occurs as a result of valance among strong electron-electron, electron-lattice, and electron-molecular correlations. Dimerization is particularly important for the physical properties of $1 \mathrm{D}$ molecular crystals because they are related to the Peierls instability to drive; relevant examples include a metal to a $2 k_{F}$-CDW insulating phase transition in a halffilled system [2] as well as the mechanism behind electronic ferroelectricity because of charge ordering in a quarter-filled system [3-7]. Learning how to control dimerization in a molecular crystal is therefore crucial for future applications to optical devices.

Potassium-tetracyanoquinodimethane (K-TCNQ) is a typical target material that can be evaluated for such a study because it is a 1D chain compound comprising stacked planar TCNQ molecules [8,9]. The chemical formula of TCNQ and the crystal structure of K-TCNQ are shown, respectively, in Figs. 1(a) and 1(b) based on referenced X-ray data [9]. This compound exhibits a magnetic-nonmagnetic phase transition with changes in the dimerization of TCNQ in the stack [10]. The phase transition is actually a type of spin-Peierls phase transition; however, it is called a "generalized" Peierls phase transition because it is a first-order phase transition demonstrating a high transition temperature $\left(T_{c}=395 \mathrm{~K}\right)$ [11]. Figures $1(\mathrm{c})$ and $1(\mathrm{~d})$ show the schematic structures of the high-temperature Mott insulating phase and

*tishi@ chem.titech.ac.jp the low-temperature dimerized (DM) phase, respectively. The intermolecular distances between the nearest neighboring TCNQs are uniform in the Mott phase but alternate to create a dimer structure in the DM phase. The DM phase is therefore attractive for investigating how to control the degree of dimerization with external fields (e.g., by light irradiation [1114] or an electric current $[15,16])$. The extremely high $T_{c}$ suggests that applications based on this phenomenon could operate at room temperature. During the last decade, this photoinduced phenomenon, created using an ultra-short pulsed laser (approximately $100 \mathrm{fs}$ ), has been enthusiastically studied by several groups. One of the most comprehensive studies, conducted by Ikegami et al. [11], was a pump-probe optical study using a 120 -fs pulsed laser. They explained the dynamics of the PIPT at hand based on a reflectivity change correlated to the charge-transfer gap. The initial photoirradiation, however, was found to instantly create a small polaronic state within their employed time resolution of $180 \mathrm{fs}$. This small polaronic state ultimately weakened the dimerization of the TCNQ and resulted in a photoinduced undimerized state at least $0.4 \mathrm{ps}$ after photoirradiation. This photoinduced undimerized state subsequently transitioned to the initial DM state with a relaxation time of approximately $3 \mathrm{ps}$. Moreover, an additional pump-probe study was also conducted by Nakata et al. [13], which likewise employed an extremely ultra-short laser pulse. They observed the frequency shift dynamics of the coherent oscillation of associated intramolecular vibrations and insisted that their obtained results reinforced those of Ikegami et al. as far as the timescale of the dimerization melting process was concerned.

Studying the dynamics of the change in dimerization is the key to clarifying the dynamics of PIPT in K-TCNQ. However, no direct observations have yet been reported. In molecular crystals, an observation of the electron-molecular vibration (emv)-coupled mode is known to be a sensitive probing of the molecular dimerization $[17,18]$. Totally symmetric $a_{g}$ 
(a)

(b)
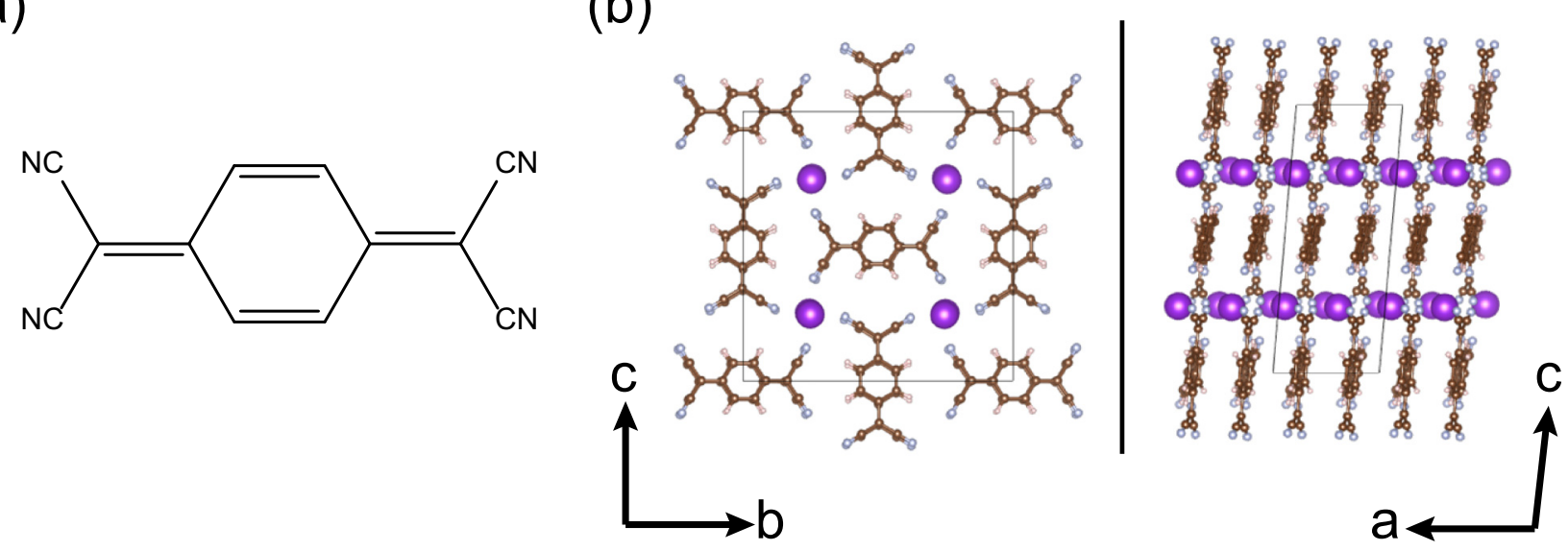

(c)

$T>T_{\mathrm{c}}:$ Mott insulator

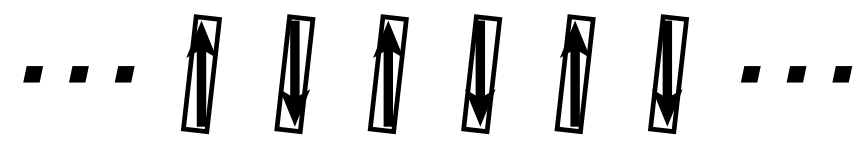

(d)

\section{$T<T_{\mathrm{c}}$ : spin-Peierls phase}
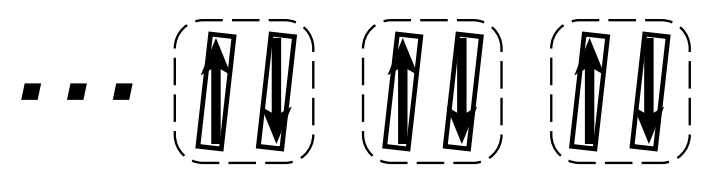

FIG. 1. (a): Chemical formula of TCNQ. (b): The crystal structure of K-TCNQ at $25^{\circ} \mathrm{C}$ [9]. The purple (online) balls represent the K atom. The thin lines represent the unit cell. (c) and (d): Schematic view of the TCNQ chain at a temperature $(T)$ above the transition temperature $\left(T_{c}\right)$, and $T<T_{c}$. The rectangles and arrows represent the TCNQ molecules and electrons with spin, respectively. The rounded and dashed rectangles represent the dimerization pairings.

modes are intrinsically IR inactive, but emv-coupled modes of them are IR active with polarization that is parallel to the molecular stacking axis. Figure 2(a) schematically depicts atomic displacement of the $\mathrm{CN}$ stretching mode, which has an $a_{g}$ symmetry. Figure 2(b) shows the temperature dependence of the reflectivity band related to the emv-coupled mode of $\mathrm{CN}$ stretching. This band, however, is strongly suppressed in the high-temperature undimerized Mott phase [19]. Thus, oscillator strength is a sensitive and direct probe of the dimerization. For this study, we directly assessed the temporal variation of dimerization in the photoinduced state, by probing the oscillator strength of emv-coupled modes.

\section{MATERIALS AND METHODS}

Sample crystals were grown for this study using the standard diffusion method, which has been reported elsewhere in detail [15]. Linear reflectivity spectra without a pump pulse were measured using a Fourier transform infrared spectrometer equipped with a Cassegrain microscope objective lens. Electric fields were parallel to the $a$ axis.
The experimental setup for time-resolved infrared vibrational spectroscopy has been reported previously [20,21], hence, it is described only in brief here. The broadband mid-infrared probe-pulse (pulse duration, $120 \mathrm{fs}$; centered around $2180 \mathrm{~cm}^{-1}$; spectral width, $150 \mathrm{~cm}^{-1}$ ) was generated by optical parametric amplification (OPA) and difference frequency generation (DFG) from the output of a femtosecond Ti:sapphire regenerative amplifier operating at $1 \mathrm{kHz}$ (center wavelength, $800 \mathrm{~nm})$. The near-infrared pump pulse $(800 \mathrm{~nm})$ was obtained from a component of the Ti:sapphire amplifier output. The spot size of the probe pulse $(0.1 \mathrm{~mm} \phi)$ was much smaller than that of the pump pulse $(0.4 \mathrm{~mm} \phi)$ at the sample surface, in order to avoid inhomogeneous excitation. The probe pulse reflected from the sample was detected by a 64-channel linear MCT $(\mathrm{HgCdTe})$ array through a 19-cm polychromator. The polarizations of the pump and probe pulses were both parallel to the $a$ axis. The pulse train of the pump pulse was modulated at $500 \mathrm{~Hz}$ with an optical chopper to obtain data with a higher signal-to-noise ratio. The delay time between the pump and the probe pulses was obtained by an optical delay line. All measurements for the pump-probe reflection spectroscopy were performed at room temperature $\left(<T_{c}\right)$. 


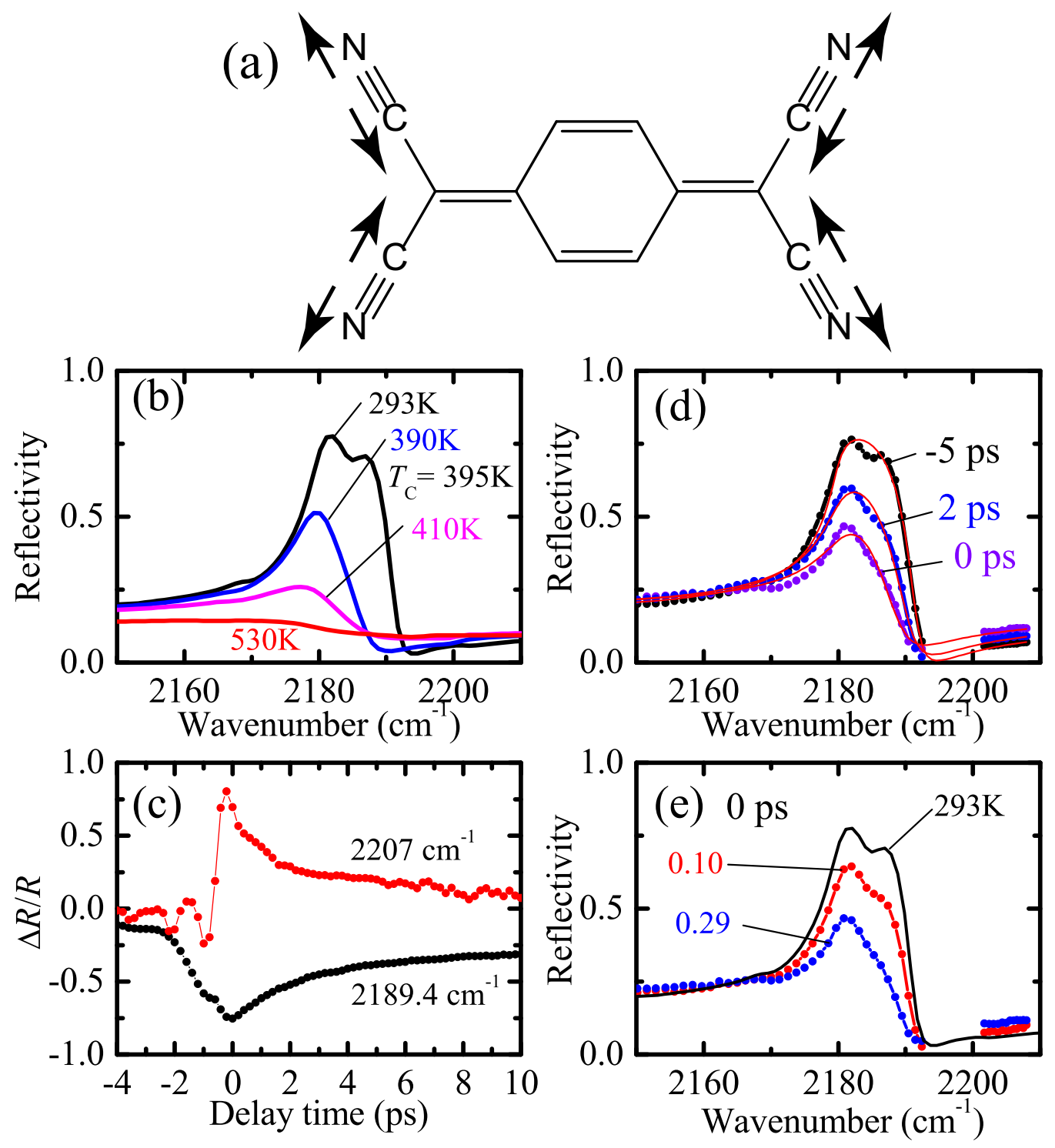

FIG. 2. (a) Schematic view of the $\mathrm{a}_{g} v_{2}$ mode. (b) Temperature dependence of the reflectivity spectra with $E \| a$ in the frequency range of the $\mathrm{a}_{g} \nu_{2}$ mode. (c) and (d) Temporal profile of $\Delta R / R$ at $2189.4 \mathrm{~cm}^{-1}$ and the photoinduced transient reflectivity spectra at certain delay times with an excitation intensity of 0.29 photon/TCNQ. The red thin lines in (d) are fitting curves. (e) The excitation intensity dependence of the photoinduced transient reflectivity spectra at time zero.

\section{RESULTS AND DISCUSSION}

\section{A. Time-resolved reflectivity measurement in the energy range of the emv-coupled mode}

We succeeded in observing the photoinduced relative reflectivity change $(\Delta R / R)$ in the mid-IR range. The typical temporal profiles of $\Delta R / R$ are shown in Fig. 2(c). As is clearly seen, it was observed that $\Delta R / R$ existed in the negative time range before time-zero. $\Delta R / R$ reached a maximum at time zero, with relaxation behavior ultimately observed during the positive time range.

During the negative time range, however, an oscillatory structure and a long-tailed slope decaying to a more negative delay was observed in several cases. The resonant frequency of the emv-coupled $\mathrm{CN}$ stretching mode was found to be $2180 \mathrm{~cm}^{-1}$. Therefore, the case of a $2189.4 \mathrm{~cm}^{-1}$ probe light corresponded to a near-resonant condition, while a $2207 \mathrm{~cm}^{-1}$ light case corresponded to an off-resonant condition. $\Delta R / R$ in the negative time range can be attributed to the signal of the perturbed free precession decay (FPD) of probelight-induced polarization, which has a long dephasing time ( ps order) [22,23]. An in-depth discussion concerning the perturbed FPD signal is provided below in Appendix A. Because the initial photoexcitation cannot directly affect the dynamics of the intramolecular oscillation, the observation of the perturbed FPD signal clearly suggests a strong interaction between the electronic structure and the emv-coupled mode. As such, we hence focus upon $\Delta R / R$ in the positive time range, in which the photoinduced dynamics of the dimerization change should be observed and not affected by the perturbed FPD phenomenon due to causality. Here, considering a certain degree of ambiguity with respect to the time-zero point because of the perturbed FPD, we wish to avoid the discussion of very early dynamics during the creation of the photoinduced state. 
In order to clarify the nature of the photoinduced state, the reflectivity spectra at different delay times were plotted in Fig. 2(d). The reflectivity band of the emv-coupled mode is clearly suppressed by photoirradiation, and the relaxation process is the recovery of the reflection band. Figure 2(e) portrays excitation fluence dependency with the reflectivity spectrum. The suppression of the reflection band becomes stronger with an increase in fluence. Comparing the zerops spectrum in Fig. 2(d) with the $390 \mathrm{~K}$ spectrum, hightemperature phase in Fig. 2(b), the bandwidth of the zero-ps spectrum is apparently more broad, while the heights are quite comparable to each other.

\section{B. Fitting analysis of the reflectivity spectra of the photoinduced state and its fluence dependency}

We employed a fitting procedure using the simple Lorentz oscillator model. In Fig. 2(b), the 293 K spectrum shows a small dip on the top of the reflection band. It was assumed that the dip structure was due to the antiresonance effect of a very weak oscillator overlapping the emv-coupled mode, which has strong oscillator strength borrowed from its charge-transfer excitation $[17,18]$. Therefore, only one Lorentz oscillator was employed for the fitting, neglecting the contribution of such a weak oscillator. The formula of the dielectric constant as a function of photon energy $(\hbar \omega)$ for the fitting was deployed as follows:

$$
\epsilon(\omega)=\epsilon_{\infty}+\frac{4 \pi \beta \omega_{0}^{2}}{\omega_{0}^{2}-\omega^{2}-i \Gamma \omega} .
$$

In this formula, $\epsilon_{\infty}$ is the constant term, and $\beta, \omega_{0}$, and $\Gamma$ are the oscillator strength, resonant frequency, and damping constant of the emv-coupled mode, respectively. The associated fitting curves are plotted in Fig. 2(d) as thin red lines and reproduce the experimental data very well. For comparison, our study also performed a fitting analysis for temperature-dependent data. The results for both the temperature dependence of the static spectra and the fluence dependence of the photoinduced spectra (at $0 \mathrm{ps}$ ) are plotted with a reflectivity value of $2180 \mathrm{~cm}^{-1}$ in Figs. 3(a)-3(f).

As for the thermally-induced phase transition, the reflectivity value gradually decreases with increasing temperature and then suddenly drops at $T_{c}$ [Fig. 3(a)]; $\beta$ also decreases with temperature and remains at a certain value, even in the high-temperature phase [Fig. 3(b)], and $\Gamma$ ultimately increases and displays a sudden rise at $T_{c}$ [Fig. 3(c)]. These behaviors imply the occurrence of the generalized Peierls transition and the simultaneous melting of dimerization. The finite value of $\beta$ in the high-temperature phase suggests that dimerization persists even though x-ray measurement shows no such dimerization [10], which is consistent with previous experimental [19] and theoretical [24] works suggesting "fluctuations" in dimerization.

The fluence dependency of the fitting parameters displays a continuous change with increase in excitation density. The direction of the parameter change can be explained by photoinduced melting as well as fluctuations in dimerization. We could not observe any sign of saturation within the fitting parameters for the evaluated fluence range. In addition, the absolute values of the changes in each parameter are smaller

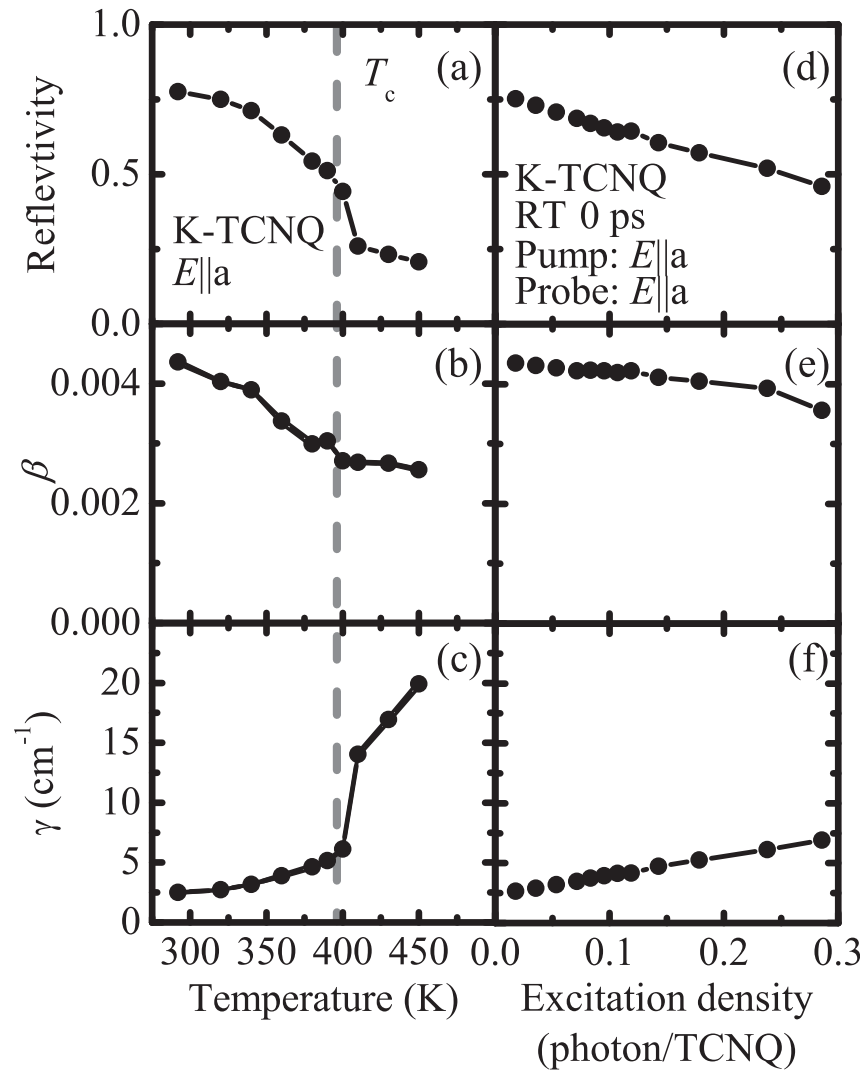

FIG. 3. Temperature dependence of (a) the value of reflectivity at $2180 \mathrm{~cm}^{-1}$, (b) oscillator strength $(\beta)$, and (c) damping constant $(\Gamma)$. The vertical dashed line represents $T_{c}$. The excitation intensity dependence of (d) reflectivity at $2180 \mathrm{~cm}^{-1}$, (e) $\beta$, and (f) $\Gamma$.

than those of the thermal phase transition case, which is consistent with a lack of saturation and suggests an imperfect phase change. Conversely, Ref. [11] reported saturation of the $\Delta R / R$ signal, which was likely attributable to the space-filling effect, even though the range of the excitation density was similar. This apparent contradiction may be partly attributed to the imperfect melting of the dimerization due to the lack of a 3D long-range order, in contrast with the perfect melting of the dimerization in Na-TCNQ discussed in Ref. [14]. The observation of a photoinduced change in oscillator strength must be correlated with a direct probe of dimerization rather than that of a change in CT gap energy. Thus, it is quite possible that there was another mechanism in play that saturated the $\Delta R / R$ signal at the probe energy they observed.

\section{Relaxation dynamics of the photoinduced state}

Figures 4(a)-4(c) show the temporal dependence of the fitting parameters in the positive time range. Among the three parameters, $\beta$ and $\omega_{0}$ show very rapid relaxation just after time zero, however $\Gamma$ does not show any rapid relaxation at all. The relaxation time constant of each parameter can be assessed by fitting analysis per the following exponential-decay-type function:

$$
f(t)=A \exp \left(-\frac{t-t_{0}}{\tau}\right)+B
$$




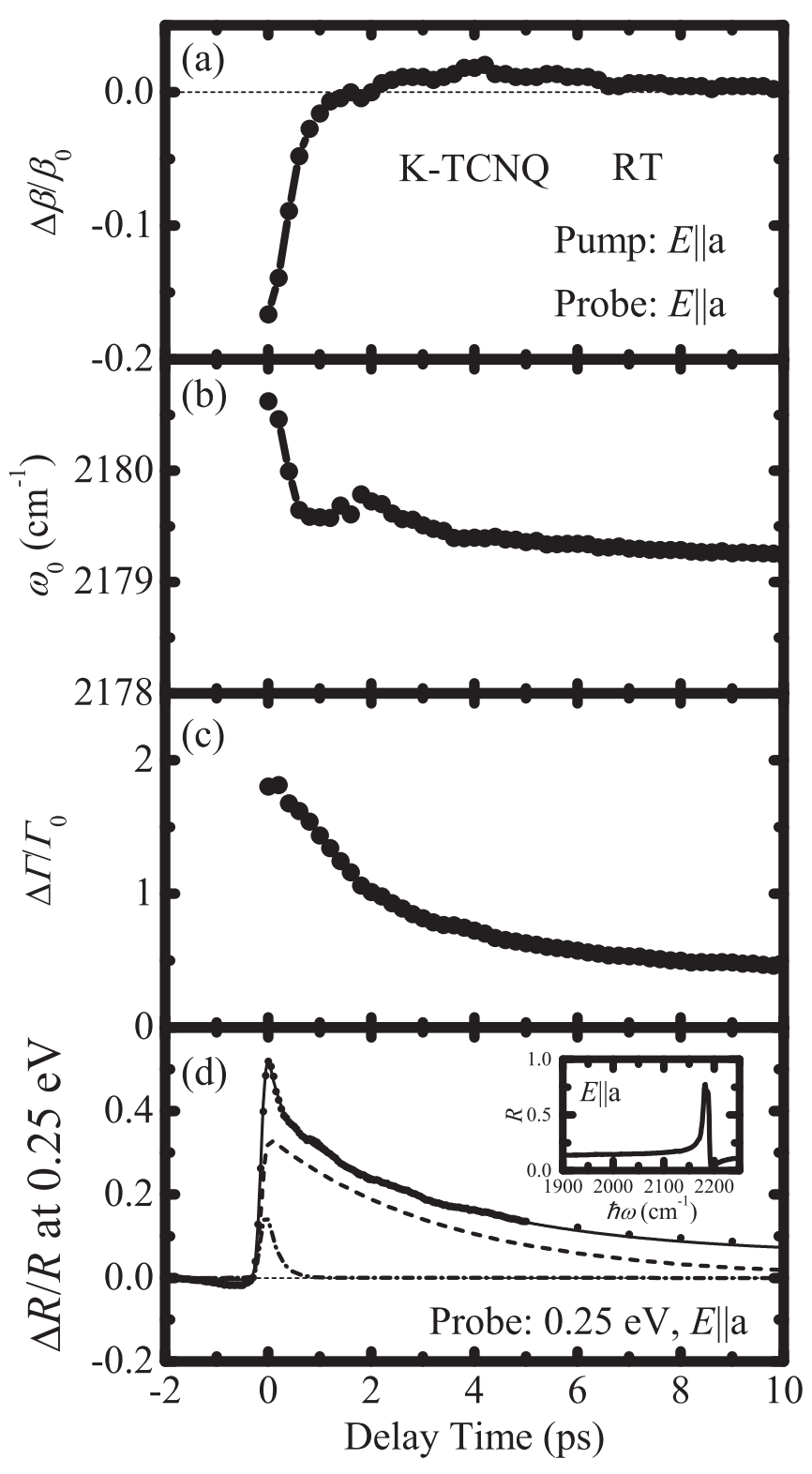

FIG. 4. Time dependence of the fitting parameters. (a) The relative change in $\beta$, (b) $\omega_{0}$, and (c) $\Gamma$. (d) Time dependence of the relative reflectivity change at $2016 \mathrm{~cm}^{-1}$ (closed circles) with the results of the fitting. The dashed lines are the components of the fast and slow relaxation (see text). Inset of (d): Reflectivity spectrum of K-TCNQ at RT with $E \| a$.

where $A$ and $B$ are constants, $t_{0}$ is time zero, and $\tau$ is the relaxation time constant. The obtained values of $\tau$ are about $0.6 \mathrm{ps}$ for $\beta, 0.7 \mathrm{ps}$ for $\omega_{0}$, and $2.3 \mathrm{ps}$ for $\Gamma$. $\beta$ must be a direct measure of dimerization, and $\omega_{0}$ should be shifted from the value of the isolated molecule that is proportional to the energy shift of the local CT gap, which ultimately changes with the melting and recovery of the dimerization [11]. Therefore, the close relationship between $\beta$ and $\omega_{0}$ is reasonable from the viewpoint of PIPT. A value of $0.6-0.7$ ps for $\tau$ of $\beta$ and $\omega_{0}$ is apparently shorter than the previously reported value of approximately $3 \mathrm{ps}$ for the recovery of dimerization melting [11]. However, we believe that the new estimated value is consistent with the time dependence of the frequency shift of the coherent intramolecular vibration phenomenon reported by Nakata et al. [13]. They had reported the photoinduced dynamics to be within $1.8 \mathrm{ps}$, although it appears they had likely stimulated the intramolecular excitation level judging from the polarization of the pump light. The photoinduced frequency shift seems to relax to its static value within $1 \mathrm{ps}$, which is similar to this present study's $\tau$ value of recovery of dimerization. In contrast, however, $\tau$ of $\Gamma$ is apparently longer than that of the other fitting parameters. It is therefore helpful to compare the temporal profile with that of the response of the electronic structural change at hand. Figure 4(d) shows the temporal profile of $\Delta R / R$ at $2016 \mathrm{~cm}^{-1}$ (about $0.25 \mathrm{eV}$ ), which is believed to be the electronic response due to there being no sign of molecular vibration in the spectrum [see the inset of Fig. 4(d)]. This photon energy is thus the same as the probe energy in Ref. [11], which was believed to detect the photocarrier response. The temporal profile of $\Delta R / R$ at $2016 \mathrm{~cm}^{-1}$ can hence be fitted with a linear combination of two exponential-decay-type functions. The obtained $\tau$ values are 0.2 and $3.1 \mathrm{ps}$, which are similar to the values for $\beta$ and $\Gamma$ of the emv-coupled mode, respectively. The increase in reflectivity suggests the creation of charged particles, such as free carriers and/or small polarons, by photoirradiation. In Ref. [11], the authors identified the charged species as "a small polaron that is created up to 0.4 ps after photoirradiation." However, they did not discuss the lifetime of the polaron precisely because the temporal profile was not able to be reproduced with a single component of the exponential decay function. In this present study, however we found an apparent correspondence between the $\tau$ of the fitting parameters of the emv-coupled mode and the electronic response. Therefore, it is believed that there are at least two types of relaxation processes for charged species in photoinduced dynamics. For the fast relaxation component of $0.2 \mathrm{ps}$, this corresponds to the recovery process of dimerization, where it can be assumed that a relaxation in the number of undimerized TCNQs is prevalent. However, the slow relaxation component of 3.1 ps can be explained by another mechanism, specifically by the fluctuation in the emvcoupled mode, because the relaxation process corresponds to that of $\Gamma$. One of the most plausible fluctuation mechanisms is the directional fluctuation of dimerization. During the relaxation process from the undimerized to the dimerized state, there is a degree of freedom for molecular pairing; dimerization can thus occur with the left or right molecule. This degree of freedom results in the creation of a domain wall soliton, which is reported to be able to move along the chain and to possess a negative charge [12]. We believe that the slow relaxation condition is actually a relaxation in the number of domain walls, in other words the creation of a single domain in the dimerized state.

\section{CONCLUSIONS}

The results of the study are schematically summarized in Figs 5(a)-5(d) below. We excited the charge transfer with 800-nm light in the DM phase [Fig. 5(a)] and directly observed the melting of the dimerization of TCNQ molecules [Fig. 5(b)], which is consistent with previously reported results based on observations of charge-transfer peak temporal dependence [11]. However, we could not observe any saturation 


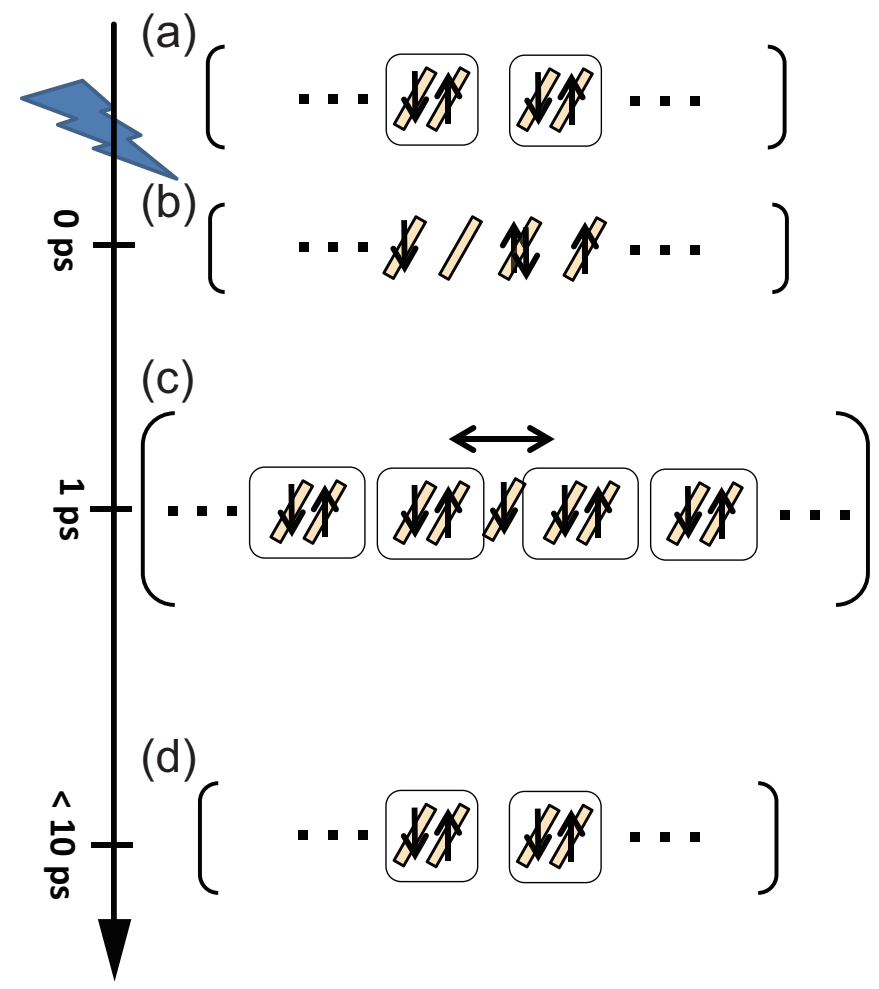

FIG. 5. Schematic photoinduced dynamics. In each image, the rectangles are TCNQ and the arrows are electrons. Rounded and dashed rectangles surrounding the molecules represent the pairing of the dimerization of TCNQ.

behavior, and the portion of the photoinduced state is therefore determined as smaller than that previously reported. The observed relaxation dynamics of the photoinduced undimerized state are different from those in previously reported cases. The oscillator strength recovers with a relaxation constant of approximately $0.6 \mathrm{ps}$, suggesting a faster recovery of dimerization than that previously reported. However, there remains a certain degree of disorder, which maintains a large value for the damping constant. This may ultimately hinder the pairing for dimerization as shown in Fig. 5(c). Such a hindered state with a mobile domain wall soliton relaxes to a large domain (accompanying the disappearance of the domain wall) with a time constant of approximately 2 ps [Fig. 5(d)], which is similar to the reported value of dimerization recovery [11]. We believe that the direct observation of dimerization dynamics has unveiled its true behavior, which could not be definitively clarified using the other possible experimental techniques because they only detect the indirect structural information via electronic structural changes.

\section{ACKNOWLEDGMENTS}

We would like to thank Mr. Sebastian Walfort for his assistance with our time-resolved optical measurements. This research was partially supported by PRESTO, JST and CREST, JST. This research was also partially supported by JSPS KAKENHI Grants No. JP15H02103 and No. JP16K05397.

\section{APPENDIX: A PHENOMENOLOGICAL DESCRIPTION OF PERTURBED FREE PRECESSION DECAY (FPD) IN THE REFLECTIVITY SPECTRUM}

The perturbed free precession/induction decay (FPD/FID) signal in the negative time range has been studied by several groups (Refs. [22,23]). However, all of the experiments performed have been measurements of transmission spectra, with no measurements being found for reflectivity spectra, except for a quantum dot system (Ref. [25]). Usually, a perturbed FPD signal is explained as a result of interference between the incident light and the radiation from induced polarization, which has a long coherence time. However, in the case of the reflectivity spectrum, we cannot consider such an interference effect because there is no incident light in play. As for the quantum dot system, the authors have considered the interference between the reflection from the sample surface and the induced polarization in the quantum dot. It was accordingly determined that the observation of the perturbed FPD signal in the bulk reflection signal was not apparent. However, it was found that one can readily observe a perturbed FPD signal even in the reflectivity spectra. In the discussion below, an expression for a perturbed FPD signal in the reflectivity spectra is hence presented and evaluated.

The following description is based on a formalism developed in Refs. [22,23]. Thus, the intent is to follow their definitions and describe only the differences from the description provided in Ref. [23]. The difference must come from the experimental setup of the measurement. As such, we detected reflected light from their associated sample due to the radiation of the probe-light-induced polarization, $E_{r}$. In the direction of the reflection of light, there was no other radiation apart from that of the induced polarization. Thus, the difference between Ref. [23] and our description starts at Eq. (1.3) in Ref. [23].

Within linear optics, the Fourier component of the radiation from the induced polarization $E_{r}(\omega)$ can be written using the Fourier components of the electric field of the incident light $E_{i}(\omega)$ and the response function $M(\omega)$ as follows:

$$
E_{r}(\omega)=M(\omega) E_{i}(\omega) .
$$

The intensity recorded by a frequency-resolved detector is

$$
I(\omega)=\left|E_{r}(\omega)\right|^{2} .
$$

Hence, the pump-probe signal has to be described by the following:

$$
\Delta_{\tau} I(\omega)=\left|E_{r, \tau}(\omega)\right|^{2}-\left|E_{r}(\omega)\right|^{2},
$$

where $E_{r, \tau}$ is the change in radiation by the pump light which reaches the sample with a delay of $\tau$ from the probe light. Following Ref. [23], we also introduce the function; $G_{\tau}(t)$ :

$$
G_{\tau}(t)=\left[E_{r}(t)-E_{r, \tau}(t)\right] / E_{r}(t) .
$$

Using this function, we can modify Eq. (A3) as follows:

$$
\begin{aligned}
\Delta_{\tau} I(\omega)= & \left|\left\{M(\omega) E_{i}(\omega)\right\} * G_{\tau}(\omega)\right|^{2} \\
& -2 \operatorname{Re}\left[\left\{M(\omega) E_{i}(\omega)\right\}^{*}\left\{\left\{M(\omega) E_{i}(\omega)\right\} * G_{\tau}(\omega)\right\}\right] .
\end{aligned}
$$


Here, $A(\omega) * B(\omega)$ is defined as the convolution of $A(\omega)$ and $B(\omega)$. However, we need to assess $\Delta_{\tau} R(\omega)$ instead of $\Delta_{\tau} I(\omega)$, and then ultimately calculate $\Delta_{\tau} R(\omega)$ :

$$
\begin{aligned}
\Delta_{\tau} R(\omega)= & \Delta_{\tau} I(\omega) / I_{i}(\omega) \\
= & \frac{\left|\int_{-\infty}^{\infty} d \omega^{\prime} M\left(\omega^{\prime}\right) E_{i}\left(\omega^{\prime}\right) G_{\tau}\left(\omega-\omega^{\prime}\right)\right|^{2}}{\left|E_{i}(\omega)\right|^{2}} \\
& -2 \operatorname{Re}\left[\frac{M^{*}(\omega) \int_{-\infty}^{\infty} d \omega^{\prime} M\left(\omega^{\prime}\right) E_{i}\left(\omega^{\prime}\right) G_{\tau}\left(\omega-\omega^{\prime}\right)}{E_{i}(\omega)}\right] .
\end{aligned}
$$

For the simplified case of $\delta$-shaped excitation and probing pulses, we obtain the following:

$\Delta_{\tau} R(\omega)=\left|M(\omega) * G_{\tau}(\omega)\right|^{2}-2 \operatorname{Re}\left[M^{*}(\omega)\left\{M(\omega) * G_{\tau}(\omega)\right\}\right]$.

For a Lorentzian-shaped absorption line (homogeneously broadened, phase relaxation time: $\left.T_{2}\right), M(t)$, is defined as

$$
M(t)=\Theta(t) e^{-t / T_{2}} e^{-i \omega_{0} t},
$$

where $\Theta(t)$ is the Heaviside function and $\omega_{0}$ is the center frequency of the absorption line. In addition, we assume the function $G_{\tau}(t)$ to be

$$
G_{\tau}(t)=C I_{p} \Theta(t-\tau),
$$

where $C$ is a constant and $I_{p}$ is the intensity of the pump light.

Using Eqs. (A8) and (A9), we can then hence modify Eq. (A7) as

$$
\begin{aligned}
\Delta_{\tau} R(\omega)= & \frac{|C|}{(2 \pi)^{2}} \frac{|M(0)|^{2}}{\left(1 / T_{2}\right)^{2}+\left(\omega_{0}-\omega\right)^{2}} \\
& \times\left[|C| I_{p}^{2} e^{-2 \tau / T_{2}}-2 I_{p} e^{-\tau / T_{2}} \cos \left\{\left(\omega_{0}-\omega\right) \tau+\delta\right\}\right] .
\end{aligned}
$$

The obtained perturbed FPD signal is therefore the summation of the exponential-decay-type function and the decayed oscillator, whose relaxation times are determined by $T_{2}$. The final term of Eq. (A10) apparently oscillates with detuning frequency.

\section{APPENDIX B: THE PERTURBED FPD SIGNAL IN THE NEGATIVE DELAY-TIME RANGE OBSERVED IN K-TCNQ.}

We have observed the pump-probe signal in the negative delay-time range as shown in Fig. 2(b). As for the time-zero point in Fig. 2(b), we approximately determined its position by substituting the sample with a silicon ( $\mathrm{Si}$ ) plate and measured the instant transmission change due to photocarrier generation in the plate itself. Figure 6 shows the probe photon energy dependence of the temporal profiles for the pump-probe signal; associated oscillating structures appear to be in play under this condition. In order to clarify the origin of the oscillations as well as the signal in the negative time range, we analyzed the oscillation frequency by "fitting" the temporal profile via the use of an exponentially decaying cosine function. Figure 7

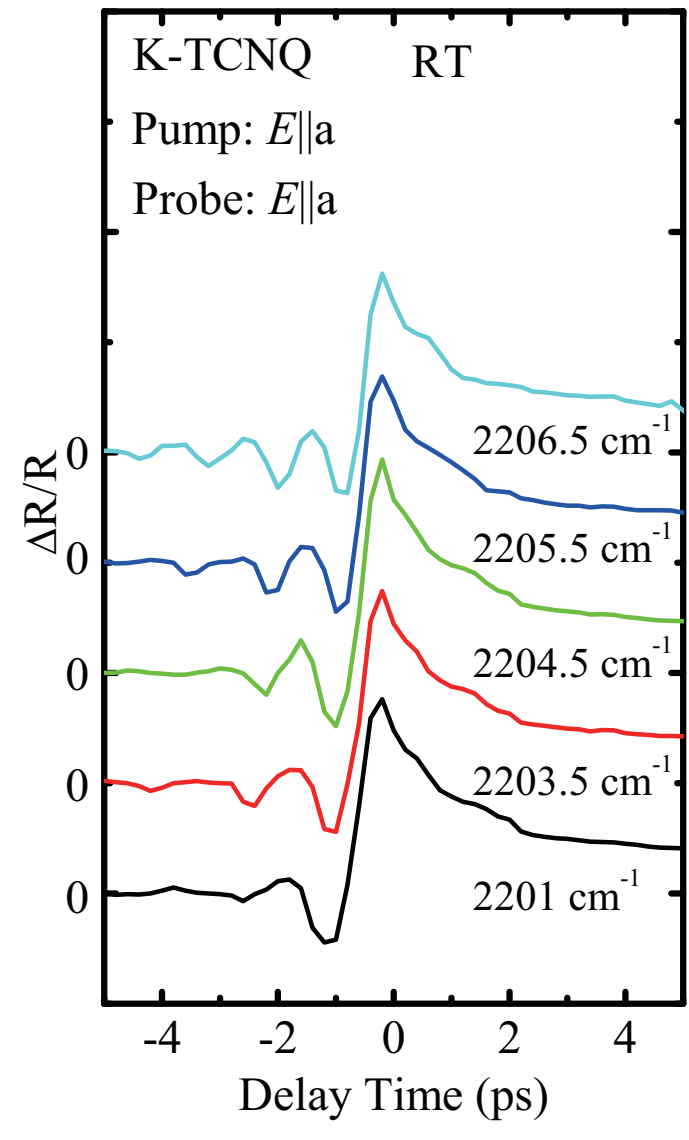

FIG. 6. Time dependence of the pump-probe signal in the negative delay time.

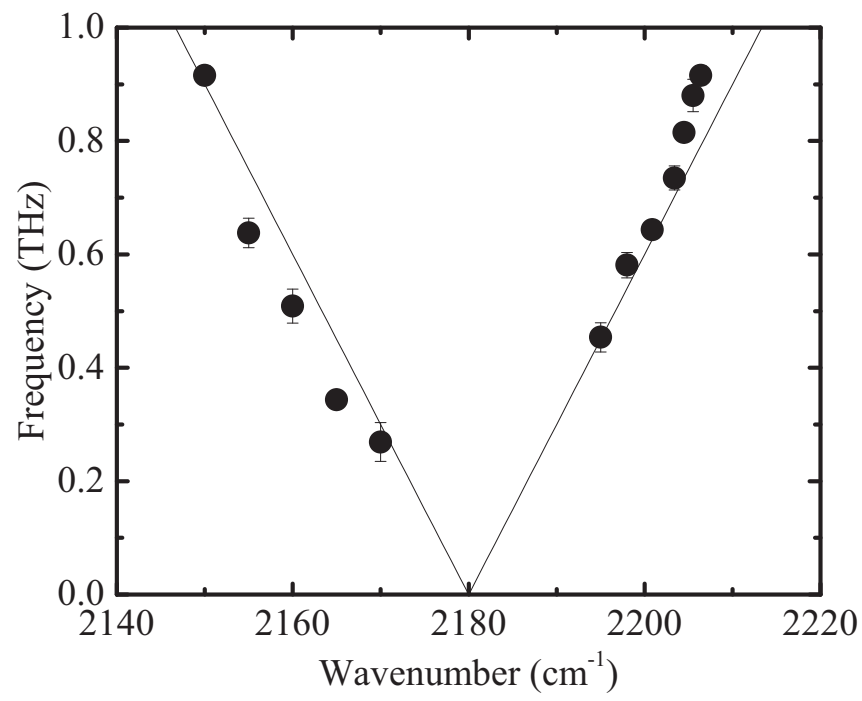

FIG. 7. Probe photon energy dependence of the oscillation frequencies in the negative delay time. The thin solid lines are the expected photon energy dependencies of the frequency, assuming a perturbed FPD. 
shows the resulting obtained frequencies in the negative time range. The solid lines portray the detuning value from the resonant frequency of the emv-coupled $a_{g}$ mode (see the main text). Moreover, the fitting parameters showed good correspondence with the detuning lines. Figure 8 shows the fluence dependency of the temporal profile for the relative reflectivity change. We ultimately examined in-depth the fluence dependency of the temporal profile; for the measurement, a Ti:sapphire laser with a pump wavelength centered at $791 \mathrm{~nm}$ was employed. In addition, we used a single photodiode to detect reflected light monochromatized by a diffractiontype $10-\mathrm{cm}$ monochromator. We believe these changes in the experimental setup did not, however, ultimately affect the observed dynamics. The pump-probe signal, both in the positive and negative delay-time range, increases linearly with increasing fluence (see the inset of Fig. 8).

These results can be qualitatively explained via the model of perturbed FPD. This is the result of a strong correlation between the emv-coupled $a_{g}$ mode and the electronic structure within this system.

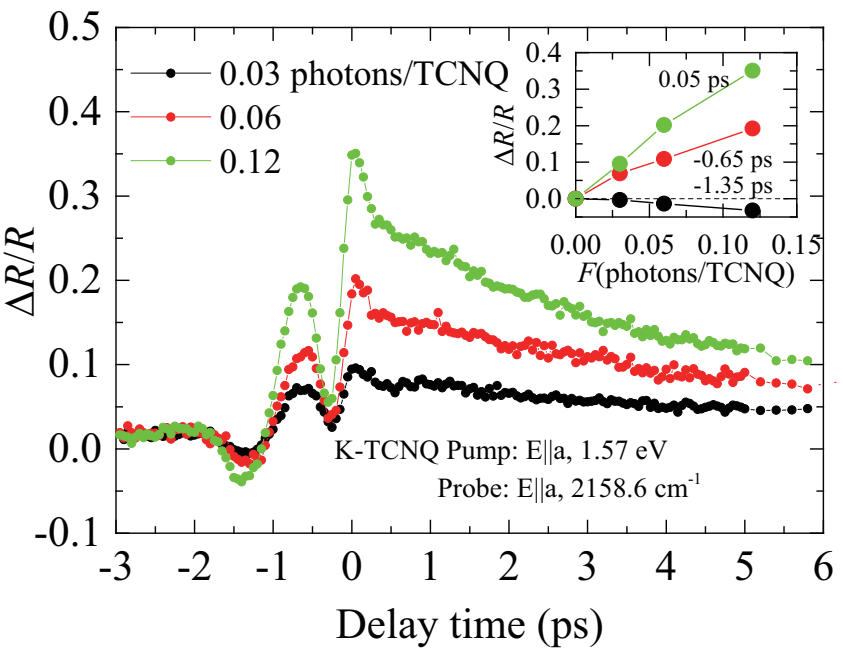

FIG. 8. Fluence dependency of the temporal profile of the relative reflectivity change in the negative delay time. Inset: Fluence dependency of the relative reflectivity change at a certain delay time.
[1] K. Nasu, ed., Photoinduced Phase Transitions (World Scientific Publishing Co. Pte. Ltd., Singapore, 2004).

[2] G. Grüner, Rev. Mod. Phys. 60, 1129 (1988).

[3] M. Abdel-Jawad, I. Terasaki, T. Sasaki, N. Yoneyama, N. Kobayashi, Y. Uesu, and C. Hotta, Phys. Rev. B 82, 125119 (2010).

[4] C. Hotta, Crystals 2, 1155 (2012).

[5] K. Kobayashi, S. Horiuchi, R. Kumai, F. Kagawa, Y. Murakami, and Y. Tokura, Phys. Rev. Lett. 108, 237601 (2012).

[6] S. Ishihara, J. Phys.: Condens. Matter 26, 493201 (2014).

[7] S. Tomić and M. Dressel, Rep. Prog. Phys. 78, 096501 (2015).

[8] M. Konno and Y. Saito, Acta Crystallogr. Sect. B 30, 1294 (1974).

[9] M. Konno, T. Ishii, and Y. Saito, Acta Crystallogr. Sect. B 33, 763 (1977).

[10] H. Terauchi, Phys. Rev. B 17, 2446 (1978).

[11] K. Ikegami, K. Ono, J. Togo, T. Wakabayashi, Y. Ishige, H. Matsuzaki, H. Kishida, and H. Okamoto, Phys. Rev. B 76, 085106 (2007).

[12] S. Koshihara, Y. Tokura, Y. Iwasa, and T. Koda, Phys. Rev. B 44, 431 (1991).

[13] K. Nakata, E. Tokunaga, J. Du, B. Xue, J. Miyazaki, K. Seto, and T. Kobayashi, Phys. Rev. B 90, 085119 (2014).
[14] H. Uemura, K. Iwasawa, H. Yamakawa, T. Miyamoto, H. Yada, and H. Okamoto, Phys. Rev. B 91, 125111 (2015).

[15] R. Kumai, Y. Okimoto, and Y. Tokura, Science 284, 1645 (1999).

[16] Y. Okimoto, R. Kumai, E. Saitoh, M. Izumi, S. Horiuchi, and Y. Tokura, Phys. Rev. B 70, 115104 (2004).

[17] A. Painelli and A. Girlando, J. Chem. Phys. 84, 5655 (1986).

[18] M. Meneghetti, Phys. Rev. B 44, 8554 (1991).

[19] H. Okamoto, Y. Tokura, and T. Koda, Phys. Rev. B 36, 3858 (1987).

[20] N. Fukazawa, T. Tanaka, T. Ishikawa, Y. Okimoto, S. Koshihara, T. Yamamoto, M. Tamura, R. Kato, and K. Onda, J. Phys. Chem. C 117, 13187 (2013).

[21] Y. Matsubara, Y. Okimoto, T. Yoshida, T. Ishikawa, S. Koshihara, and K. Onda, J. Phys. Soc. Jpn. 80, 124711 (2011).

[22] M. Joffre, D. Hulin, A. Migus, A. Antonetti, C. Benoit à la Guillaume, N. Peyghambarian, M. Lindberg, and S. W. Koch, Opt. Lett. 13, 276 (1988).

[23] P. Hamm, Chem. Phys. 200, 415 (1995).

[24] M. Kumar, B. J. Topham, R. Yu, Q. B. D. Ha, and Z. G. Soos, J. Chem. Phys. 134, 234304 (2011).

[25] T. Guenther, C. Lienau, T. Elsaesser, M. Glanemann, V. M. Axt, T. Kuhn, S. Eshlaghi, and A. D. Wieck, Phys. Rev. Lett. 89, 057401 (2002). 